\title{
Modelling of the bullet perforation of textile targets
}

\author{
R. Barauskas ${ }^{1}$, A. Abraitiene ${ }^{2}$ \& A. Vilkauskas ${ }^{1}$ \\ ${ }^{1}$ Department of System Analysis, \\ Kaunas University of Technology, Lithuania \\ ${ }^{2}$ Lithuanian Textile Institute, Lithuania
}

\begin{abstract}
The paper deals with the further development of the finite element models created in LSDYNA for simulation of the ballistic impact and perforation of the woven fabrics structures at velocities $\sim 300 \mathrm{~m} / \mathrm{s}$. The bullet has been considered as a deformable body in contact with the fabric package presented by interwoven yarn structure. The simplification of the model has been achieved by presenting the multifilament yarns by thin shell elements the thickness of which represents the real thickness of yarns as it can be measured in the weave. The model considered in earlier publications of the authors on this topic has been significantly improved by introducing an orthotropic roughly meshed membrane model for the zones of the fabric remote from the point of impact and by proper adjustment of material parameters ensuring the minimum cumulative wave propagation speed error along selected directions. A set of physical and numerical experiments including the ballistic lead bullet - lead plate and lead bullet - single woven layer interactions have been performed in order to identify the model parameters.

Keywords: high-velocity impact, multi-layer fabric, finite elements, LSDYNA.
\end{abstract}

\section{Introduction}

The rapid rise and variety of new textile materials on the market intended for life-protection clothing [2], promotes the further theoretical and experimental investigations of fabric packages by establishing their properties and regularities. The design of multilayer fabric packages (MLFP) can be significantly facilitated by means of deeper understanding of the behavior of a single or several fabric 
layers during their interaction with bullets. The results obtained from computer simulations could expedite the selection of a structure of a package designated for ballistic tests and simultaneously decrease the expenditures needed for optimization of packages made of fabrics of different surface density and different constitution.

The experimental investigations of ballistic interaction are complicated because of very large velocities, deformations and failure of interacting structures. Conventional experiments mostly provide integral characteristics of the processes such as the pre- and post-impact velocities, extent of final failure of the projectile and the target, etc. The main shortage of such kind of results is their inability to provide the insight into the interaction processes and to clarify transient dynamic processes and failure mechanisms taking place at the contact zone. A lot of research has been carried out in the field of finite element simulation of the ballistic impact on high strength fabric structures [3-7].

Finite element techniques and commercial software principally enable to model the ballistic interaction of woven textile structures with rigid or deformable bodies. Textiles used for life protecting clothing are essentially multi-scale structures made of micro-scale fibrils, which are bundled to form a yarn, which are then tightly woven into sheets. The basic limitation that prohibits direct solution of the ballistic interaction problems by employing explicit dynamic modeling is a huge dimensionality of the obtained finite element model. Most present researches on the topic employ reasonable simplifications or multi-scale approaches for the generation of a finite element model. In [5] the commercially available code LS-DYNA was used to model the ballistic impact and perforation of a rigid sphere into a patch of plain weave fabric. The yarns comprising the woven patch have been modeled as volumetric structures made of orthotropic material. Yarn-to-yarn and yarn-to-rigid body contact interaction and yarn failure criterion based on von Misses stress has been taken into account and the influence of friction on the velocity reduction of the sphere has been investigated. The mezzo-to-macro multiscale approach has been employed in [6]. The model has been based of the assumption that on the macro-scale the fabric behaves as a continuum membrane while on the mezzo-scale the properties of the structure are accounted for by a constitutive law derived by modeling a pair of overlapping crimped yarns as extensible elastic. In [7] a micro-to-mezzo multiscale approach enabled to achieve a significant reduction in the computational effort by first pre-computing the responses for a population of yarn over the entire possible deformation range, and then constructing a constitutive response with variations reflecting the approximate yarn-to-yarn differences. Such an approach enabled to model the failure of the fabric during $152 \mathrm{~m} / \mathrm{s}$ ballistic interaction.

The present work presents a further development of the deformable bullet against MLFP $\sim 300 \mathrm{~m} / \mathrm{s}$ ballistic interaction model documented earlier in [8-10]. The model has been is created by using the LS-DYNA code. Two-step multi-scale approach has been employed. Firstly, the yarns of the weave have been approximated by orthotropic shell elements, and secondly the woven yarn structure has been presented as a patch surrounded by roughly meshed 
orthotropic membrane zone in order to simulate adequately the "infinite" surrounding of the ballistic interaction zone. The two zones have been connected together by using a tied interface available in LS-DYNA. Several copies of the model of a single sheet have been positioned properly in space thus forming the model of the MLFP. Comparisons with the experimental results have been carried out in order to determine the dynamic parameters of the material behavior and to validate the model.

\section{The architecture of the model}

The overall view of the quarter symmetry model of the lead bullet and MLFP interaction is presented in Fig.1. The bullets used during the ballistic strength evaluation tests can be very different subject to the required safety level. In this study we employ the finite element model of the $6 \mathrm{~mm}$ lead bullet BALLE 22 . Material *MAT_PLASTIC_KINEMATIC is used with elasticity modulus, Poisson's ratio and density considered as prescribed. At $\sim 300 \mathrm{~m} / \mathrm{s}$ impact velocity the problem is classified as high velocity contact-impact interaction problem where the yield stress value is assumed to be dependent upon the strain rate in accordance with the Symonds-Couper material model as

$$
\sigma_{Y}=\sigma_{Y 0}\left[1+\left(\frac{\dot{\varepsilon}}{C}\right)^{\frac{1}{p}}\right],
$$

where $\sigma_{Y}, \sigma_{Y 0}$ - yield stress limits of the material defined with and without the influence of strain rate $\dot{\varepsilon} ; \mathrm{C}$ and p - constants.

Each fabric layer is made of yarns of certain linear density woven together. Each yarn consists of filaments the number of which can vary from several hundred to several thousand. The model of a fabric at the level of filaments is unrealistic because of limited computer resource, so we develop a mezzo-mechanical model in which a yarn has been considered as primary component comprising the fabric. Four shell elements the thicknesses of which were selected in order to fit two circular segments shaped cross-section have been employed across the width of the yarn. The bending stiffness of a yarn we assumed to be negligible. In order to eliminate the bending stiffness in LS-DYNA the Hughes-Liu shell element with single integration point through the thickness of the element has been used. The technique of obtaining the model of a woven yarn patch in more detail has been described earlier in our publications $[8,9]$. By using this technique the weave is obtained by solving the contact interaction equilibrium problem and is able to present the warp and weft yarns in the weave. The Ox directed yarns (the warp) are elastically deformed by moving their nodes in the direction Oz perpendicular to the plane of the layer in order to situate the Oy directed yarns (the weft) in-between the warps. After activation of the contact search algorithm the yarns of the model come to an elastic equilibrium. As a result, a woven structure is obtained in a similar way as a real fabric is produced. After the equilibrium is obtained, the elastic stresses and strains are being fully or partially removed in order to imitate the relaxation 
of stresses in multi-filament yarns. Moreover, we may control the amount of the residual stress and to create a desired value of the warp tension that is an important characteristic of a weave.



Figure 1: Quarter-symmetry model of the lead bullet-fabric package interaction.

The zones of the fabric remote from the interaction zone are modelled by means of orthotropic shell (membrane) elements that can be much larger than the elements presenting the mesh of the yarns. Sudden transitions in mesh density of finite element models are implemented by using tied interfaces. In LS-DYNA the finely and roughly meshed zones are connected by using the *CONSTRAINED_TIE-BREAK constraint. We prohibit the failure of the constraint by using very large failure strain values and no-failure material model for the yarns neighbouring to the membrane model nodes. The reason for such "numerical failure prevention" measure is that the algorithm of implementation of tied interface constraints in LS-DYNA tends to overestimate the strain values in local zones of refined mesh adjacent to the roughly meshed membrane domain. Sometimes a situation may occur when the locally overestimated strain value may cause a failure of a yarn where it appears to be not realistic physically. A uniform membrane-type surrounding of the woven patch cannot be expected to present an identical dynamic behaviour as the woven yarn structure. However, satisfactory approximation is possible provided that the appropriate selection of geometrical and physical properties of the membrane zone is made. Two models of the fabric layer with different dimensions of the woven patch are built, Fig 2 . The model containing a larger patch has been considered as a reference model (in our study it contained 120x120 yarns), and the smaller one was 20x20 yarns; we take it at least twice greater than the diameter of the zone at which the failure 
of yarns may take place. By taking into account the quarter-symmetry of the model we have to build 10x10 and 60x60 yarn woven patch models correspondingly. The size of membrane-type surrounding of the patches is much larger than the woven patches, however, the total linear dimension of each model is the same.

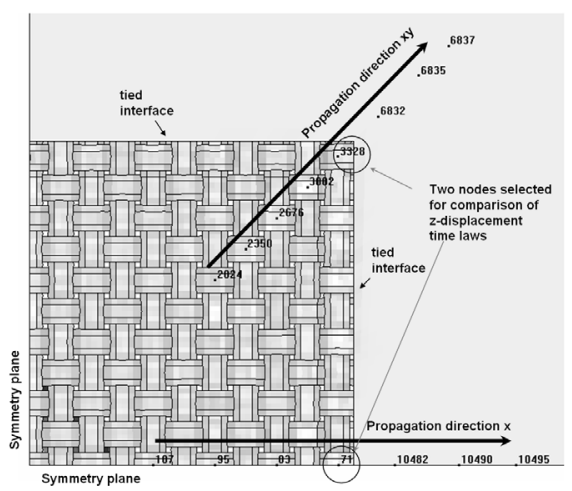

(a)

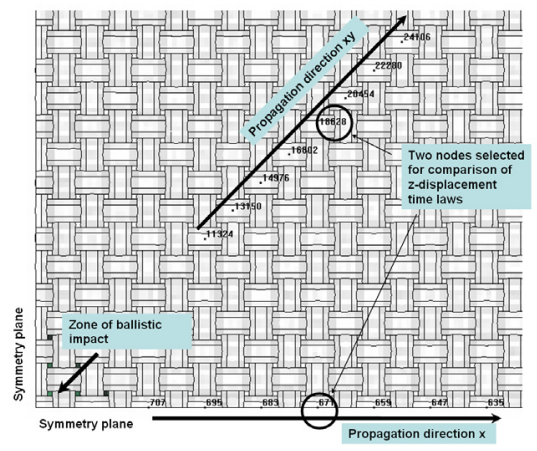

(b)

Figure 2: Fragments of two woven fabric models (a) woven patch 10x10 yarns; (b) fragment of the woven patch 60x60 yarns (reference model).

The membrane is presented by 1-integration point shell elements exhibiting no bending stiffness. The membrane thickness may be assumed as known, however, the values of mass density $\rho$, Young's modulus E and shear modulus $G$ of the orthotropic material have to selected in order to obtain the dynamic response of both models as close as possible to each other. In LS-DYNA the orthotropic material can be defined as *MAT_ORTHOTROPIC_ELASTIC or *MAT_FABRIC. The best results can be expected if the selection of parameters is accomplished for the dynamic behavior close to the specific analysis situation. In this work the numerical tests are performed for the $270-300 \mathrm{~m} / \mathrm{s}$ impact and perforation of the fabric by the lead bullet. 


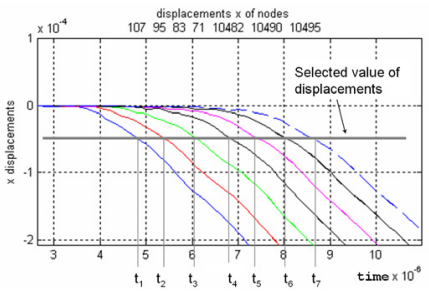

(a)

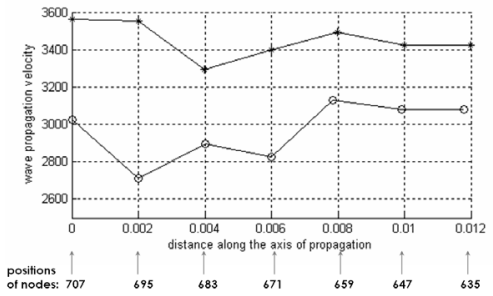

(c)

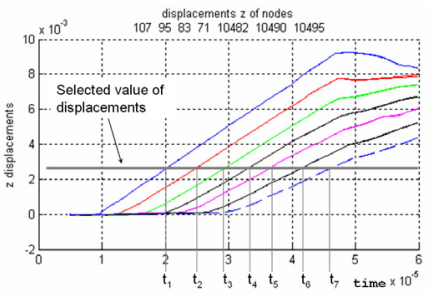

(b)

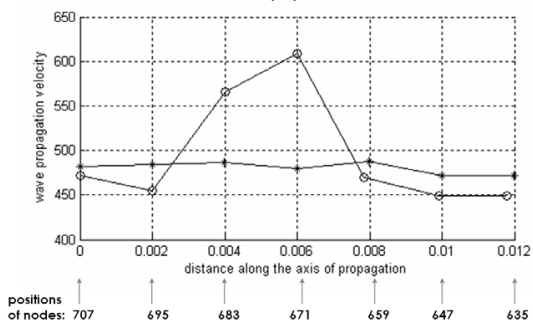

(d)

Figure 3: (a), (b)-time laws of longitudinal (a) and transverse (b) displacements of nodes and determining impact wave propagation speeds along direction $\mathrm{x}$ in the vicinity of the tied interface; (c), (d)-estimated propagation speeds of longitudinal (c) and transverse (d) waves along direction $\mathrm{x}$ in the vicinity of the tied interface: -oobtained by using model (Fig.2(a)); - *- obtained by using the reference model (Fig.2(b)).

The overall approach is similar to the convergence investigation of models with different levels of refinement. The "exact" numerical solution may be regarded as known as we are able to make a stand-alone numerical experiment by employing the fully woven model. Further a series of models with different sizes of the woven patch are investigated and the physical constants of the surrounding orthotropic membrane established in order to ensure a convergent behavior in the sense that the solutions obtained by using every model are close to the exact one. In our investigation the comparison of the dynamic behavior of the models has been performed in terms of:

- variation of the propagation speed of the longitudinal and transverse in the vicinity of the tied interface of the model; time laws of $z$ displacement of two selected nodes;

- time instant of initiation of failure of the fabric;

- time instant of full perforation of the fabric.

In order to estimate the wave propagation speed as the wave propagates in between two nodes of the structure we fix time moments $t_{1}, t_{2}$ when the displacements of the nodes reach a pre-selected level, see Fig.3(a) and (b). The longitudinal and transverse wave propagation speed estimated along direction $\mathrm{x}$ is presented in Fig.3(c),(d). The results are obtained by using one of "successful" sets of membrane material constants as 
$\frac{\text { Surface density of the membrane }}{\text { surface density of the woven patch }}=0.8 ; \frac{\text { Young's modulus of the membrane }}{\text { Young's modulus of the yarn }}=0.3$;

$\frac{\text { Shear modulus of the membrane }}{\text { Young's modulus of the membrane }}=0.0042$.

Rather small value of Young's modulus of the equivalent membrane enables to present the de-crimping driven deformation of the fabric which can be expected to prevail in zones remote from the point of impact. Very small values of shear modulus are quite natural to expect because of small shear stiffness of a woven structure at small strains. However, the value of shear modulus had to be selected with care as it makes a significant influence basically upon the shape of the front of the transverse wave, as well as, upon its amplitude. The displacements and wave fronts obtained by using 10x10 model are close to the results obtained by using the reference model as can be observed from the results presented in Fig.4.

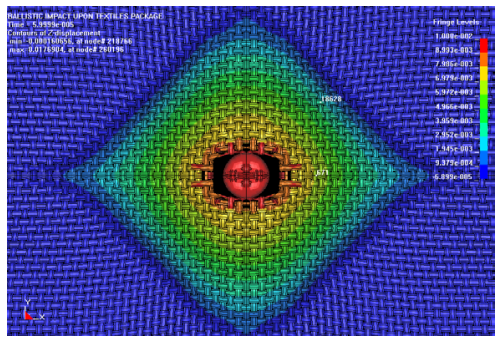

(a)



(c)

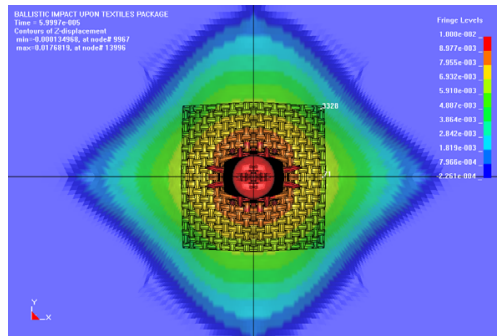

(b)

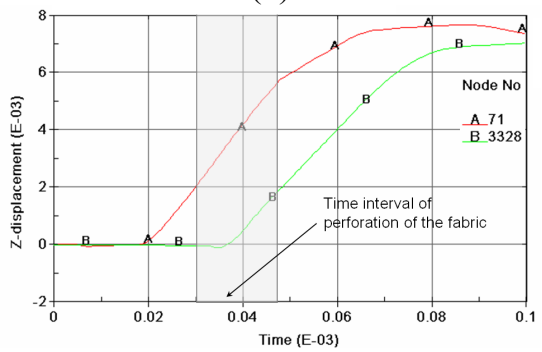

(d)

Figure 4: The shape of the transversal wave front and z-displacement contour plot obtained by using reference model (a) and 10x10 combined model (b): (a), (b)-at time instant $6 \times 10^{-5} \mathrm{~s}$ (full perforation); (c), (d) -z-displacement time laws of two selected nodes as indicated in Fig.2.

Our investigation demonstrated that it is hardly possible to select the membrane material properties ensuring very good approximation to the woven structure behaviour in all stages and aspects of its motion. For example, the model that properly represents the process of failure of the fabric may produce certain displacement errors in representing its motion after the perforation, see 
Fig.7(g), (h). On the contrary, a model adequately representing the after-perforation motion of the fabric may produce inaccuracies in estimating the time instant of the initiation of the failure. In the ballistic interaction problem the proper representation of the time moment of beginning of failure of the fabric is of primary importance, therefore by adjusting the membrane layer parameters it has been achieved that the failure begins in both models at the same time instant.

\section{Model validation and results}

The adequacy of the obtained results to reality is a highly important question in any simulation. In the case of the system under consideration main sources of possible inadequacy are the "mezzo-mechanical" concept of the model where the multi-filament structure of a thread is highly simplified, the use of the "macro-mechanical" concept for presenting the "infinite" fabric environment of the woven patch and unknown values of the dynamic parameters of materials.

The basic experiments we made in order to validate the model were as follows:

- shooting a lead bullet against the $10 \mathrm{~mm}$ thickness lead plate thus considering a problem with only one unknown material - the lead. This research has been described in detail in [10];

- shooting a lead bullet against a single fabric layer. The properties of the bullet model having been determined earlier, now we had once again a single unknown material - the paraaramid weave, friction coefficients of which could be determined;

- shooting a lead bullet against a MLFP.

The experiments provided the data enabling to quantify the correction of the paraaramid failure strain value for the range of strain rates corresponding to $\sim 300$ $\mathrm{m} / \mathrm{s}$ impact.

The bullet has been shot through a large piece of the fabric $(\sim 1 \mathrm{~m} \mathrm{x} 1 \mathrm{~m})$ so the waves excited by the impact did not reach the boundaries of the fabric during the time of the perforation. The boundary conditions imposed on the model were presented as unsupported boundaries. The data obtained from the physical experiment were the pattern of broken yarns and maximum deflection of the fabric at the time moment of full perforation.

As a base assumption we used the purely elastic paraaramid material properties to the failure limit. This is a well-known experimental fact obtained by static tests of paraaramid yarns. The values of elasticity modulus, Poisson's ratio and mass density were considered as prescribed. The elastic failure limit of the material is modeled by assuming the plastic material with failure strain 0.04 corresponding to the yield stress $3.6 \mathrm{e}+9 \mathrm{~N} / \mathrm{m}^{2}$.

The parameters values to be determined by comparing the computed results against the experimental ones are the yarn-to-yarn sliding friction coefficient FSs and bullet-to-yarn sliding friction coefficient FSk between the yarns of the woven structure and the surface of the bullet. For the sake of simplicity we didn't introduce additional parameters specifying the dependency of the friction coefficient against the velocity of sliding. 


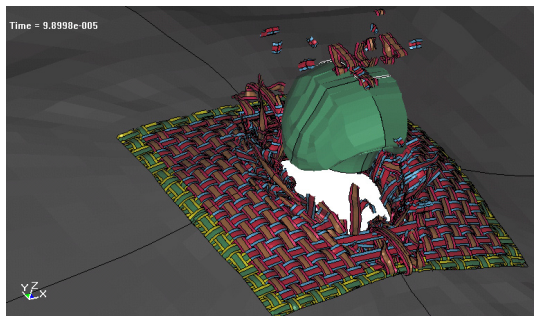

(a)

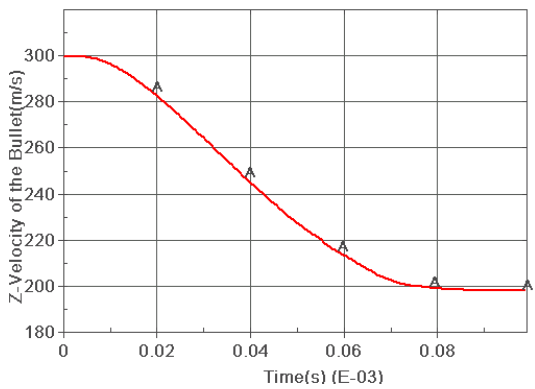

(c)

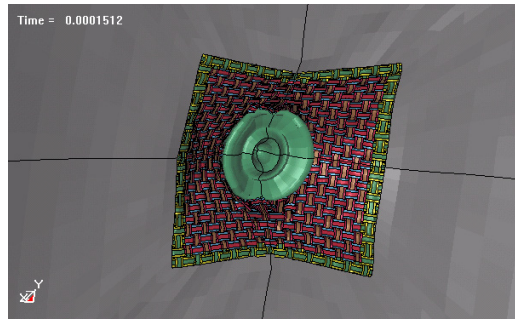

(b)



(d)

Figure 5: $\quad 300 \mathrm{~m} / \mathrm{s}$ velocity impact of a lead bullet upon a multi-layer fabric package (a), (c)-5 layers of fabric, general view of the fabric package and bullet after perforation (a) and velocity time law of the bullet (c) (b), (d)-12 layers of fabric, general view of the stopped bullet (b) and time law of the velocity of the bullet (d).

The geometrical patterns of the yarn structure at time moments at the initiation and at full perforation, corresponding transverse deflections of the fabric surface, as well as, final plastic deformations of the bullet have been investigated and compared between simulation and experiment. The comparison of the results indicated that perforation of the fabric model by breaking 4 yarns (it is the most often observed case in the reality) is possible in quite narrow range of values of friction coefficients as

$$
0.1<F S k<0.2 ; \quad 0.1<F S s<0.2 ; \quad F S s+F S s<0.3 \quad .
$$

Numerical experiments of the bullet against MLFP interaction have been performed by considering interactions between layers at zones distant from the impact point as weak because of initial clearances between the layers of the freefabric package. Comparisons with the physical experiment have been performed at 'integer' level by evaluating the ability of the package to hold up the bullet. The obtained results indicate that 10-12 layered paraaramid MLFP package is nearly a ballistic limit against a $6 \mathrm{~mm}$ lead bullet moving at $300 \mathrm{~m} / \mathrm{s}$, Fig.5. The bullet holdup effect decreases as the friction coefficient between layers is decreased (e.g., when the humidity of the environment increases). 


\section{Conclusions}

The finite element analysis of the interaction process of paraaramid multilayer fabric packages against a lead bullet has been performed in LS-DYNA by taking into account real geometries and deformability of interacting parts. The main goal of the presented study was to develop a workable model suitable for simulation of the ballistic interaction of MLFP of real dimensions by employing moderate computer resources and with satisfactory level of adequacy.

The size of the model was reduced to reasonable dimensions by presenting the yarns in the woven fabrics structure as narrow bands of a prescribed crosssection and by presenting the zones of the fabric distant from the point of impact by an orthotropic membrane model. The process of shooting-through one fabric layer has been simulated and the combined model consisting of a woven patch tied to a uniform orthotropic membrane has been verified. Comparisons of the computed results against the experimental ones have been performed in order to find the values of the dynamic material parameters of the model.

The presented research suggests prospective directions of the research of the ballistic response of free-fabric structures, such as further improvement of multifilament yarn model, ballistic interaction analysis by taking into account the biomechanical properties of human body protected by the textile packages, etc. The validation of the model is neither onetime nor fully unambiguous action. Along with improvements of the model and availability of new experimental data the perfection level of the validation is increased step by step.

The research has been sponsored by the Lithuanian Science and Studies Foundation.

\section{References}

[1] Byrne C., Davies B. New Yarn and Fiber Development Drives Technical Textiles Market. International Fiber Journal, 1999;2:100-102.

[2] Cunnif P.M. An analysis of the system effects in woven fabrics under ballistic impact. Text. Res. J., 1992; 62(9):495-509.

[3] Tan V.B.C. Lim C.T., Cheong, C.H. Perforation of high strength fabric by projectiles of different geometry. Int. J. Impact Eng., 1992; 28(2):207-222.

[4] Shim V.P.W., Tan, V.B.C., Tay T.E. Modeling deformation and damage characteristics of woven fabric under small projectile impact. Int. J. Impact Eng., 1995;16(4):585-605.

[5] Duan Y., Keefe M., Bogetti T.A., Cheeseman B.A. Modeling the role of friction during ballistic impact of a high strength plain-wave fabric. Composite Structures, 2005;68(3): 331-337.

[6] B.Nadler, P.Papadopoulos, D.J.Steigman. Multiscale constitutive modeling and numerical simulation of fabric material. Int.J.of Solids and Structures, 2006;43: 206-221.

[7] Zohdi T.I., D.Powell. Multiscale construction and large-scale simulation of structural fabric undergoing ballistic impact. Comp. Methods Appl. Mech. Engrg., 2006;195: 94-109. 
[8] A. Abraitienè, L. Valasevičiūte, R. Barauskas, A. Vilkauskas, Finite element analysis of ballistic properties of a multilayer textile package in LSDYNA, Materials Science, 2002, 8(4): 500-505.

[9] R.Barauskas, A.Abraitiene, A.Vilkauskas. Simulation of a ballistic impact of a deformable bullet upon a multilayer fabric package. 2nd International Conference on Computational Ballistics, WIT Press, Southampton, Boston, 2005: 41-51.

[10] R.Barauskas. Modeling of the bullet penetration into textile targets by using combined woven structure-membrane approach. WSEAS Transactions on Information Science and Applications, 2005, (11)2: 1944-1954. 